\title{
DIAGNÓSTICO NUTRICIONAL E ELABORAÇÃO DE MATERIAL DIDÁTICO PARA EDUCAÇÃO NUTRICIONẢL DE ESCOLARES
}

\author{
Alyne Michelle Botelho \\ Universidade Federal de Santa Catarina \\ alyne.botelho@gmail.com \\ Camila Martinelli Veiga \\ Universidade Federal de Santa Catarina \\ camilamartinelliv@gmail.com \\ Luciana Jeremias Pereira \\ Universidade Federal de Santa Catarina \\ lucianajere@hotmail.com
}

\author{
Giosana Maria Bizarro \\ Universidade Federal de Santa Catarina \\ giosananutricao@hotmail.com \\ Maria Alice Altenburg de Assis \\ Universidade Federal de Santa Catarina \\ maria.assis@ufsc.br \\ Patricia Faria Di Pietro \\ Universidade Federal de Santa Catarina \\ patricia.di.pietro@gmail.com
}

Francilene Gracieli Kunradi Vieira Universidade Federal de Santa Catarina francilene.vieira@ufsc.br

\section{Resumo}

O estado nutricional e o consumo alimentar de escolares da rede pública municipal de Florianópolis, SC, foi avaliado com o objetivo de elaborar um plano de ação organizado na forma de material didático para a educação nutricional no ambiente escolar. O estado nutricional foi avaliado pelo índice de massa corporal e o consumo alimentar, através do Questionário do Consumo Alimentar e Atividade Física de Escolares (webCAAFE). Entre março e novembro de 2014, 640 escolares de 7 a 10 anos de nove escolas da rede pública foram avaliados, sendo observados $20 \%$ de sobrepeso, $13 \%$ de obesidade e elevado consumo de alimentos não saudáveis. O material didático organizado em uma cartilha abordou oito temáticas, estruturada com texto de apoio ao professor, exemplo de aula dialogada, texto de apoio ao aluno e atividades lúdicoeducativas. Espera-se que a cartilha contribua para o incremento das ações de educação nutricional e auxilie na promoção de hábitos alimentares saudáveis desde a infância.

Palavras-chave: Consumo Alimentar. Escolares. Estado Nutricional. Educação Nutricional. Material Didático.

\section{NUTRITIONAL DIAGNOSIS AND DEVELOPMENT OF TEACHING MATERIALS FOR NUTRITION EDUCATION OF SCHOOLCHILDREN}

Abstract

The nutritional status and food intake of school-aged children from the municipal public school network of the city of Florianopolis, SC, were evaluated in order to draw up a plan of action organized in the form of educational materials for nutrition education at school. The nutritional status was assessed through the Body Mass Index (BMI) and food consumption through the online Questionnaire on Food Consumption and Physical Activity in School-Aged Children (webCAAFE). Between, march to november of 2014, 640 school-going children aged 7 to 10 years from nine municipal public schools were evaluated. A prevalence of $20 \%$ of overweight and $13 \%$ of obesity was observed, in addition to a high consumption of foods of low nutritional value. The teaching material was organized in a booklet covering eight different subjects, structured with supporting text for the teacher, an example of a collaborative class, supporting text for the student and playful educational activities. It is hoped that the booklet will contribute to the improvement of nutritional education actions and assist in the promotion of healthy eating habits since childhood.

Keywords: Food Intake. Schoolchildren. Nutritional Status. Nutrition Education. Courseware.

\section{DIAGNÓSTICO NUTRICIONAL Y PREPARACIÓN DE MATERIAL EDUCATIVO PARA LA NUTRICIÓN DE LA EDUCACIÓN ESCOLAR}

Resumen

El estado nutricional y el consumo alimentar de niños en edad escolar de Florianópolis, SC, fue evaluada con el fin de elaborar un plan de acción organizada en forma de materiales educativos para la educación nutricional en la escuela. El estado nutricional se evaluó mediante el índice de masa corporal y la ingesta dietética por la encuesta de la ingesta de alimentos y la Actividad Física de la Escuela (webCAAFE). Entre marzo y noviembre de 2014, se evaluaron 640 alumnos de 7 a 10 años a partir de nueve escuelas públicas y se observaron $20 \%$ del exceso de peso, el 13\% de la obesidad y el alto consumo de alimentos poco saludables. El material de instrucción organizada en un folleto dirigido ocho temática de texto, estructurado con apoyo del maestro, ejemplo de la clase dialógica, apoyo a los estudiantes texto y las actividades recreativas y educativas. Se espera que el folleto contribuirá al aumento de la educación sobre la nutrición y ayudar en la promoción de hábitos alimentarios saludables desde la infancia.

Palavras clave: Consumo de Alimentos. Escuela. Estado Nutricional. Educación Nutricional. Material Didáctico 


\section{INTRODUÇÃO}

No Brasil, a transição nutricional tem ocorrido rapidamente, caracterizada pela redução dos casos de déficits nutricionais e aumento da prevalência de sobrepeso e obesidade não só na população adulta, mas também em crianças e adolescentes (MATOS et al., 2011; BATISTA FILHO et al., 2008). Segundo a Pesquisa de Orçamentos Familiares (POF 2008-2009), o excesso de peso (sobrepeso e obesidade) atinge hoje um percentual de 51,4\% e 43,8\%, de meninos e meninas, respectivamente, entre 5 e 10 anos de idade (IBGE, 2010).

Além disso, observam-se também mudanças nos padrões alimentares como o aumento no consumo de guloseimas (bolachas recheadas, salgadinhos, doces) e refrigerantes (TRICHES; GIUGLIANI, 2005). Estudo realizado com 2.936 escolares de 7 a 10 anos de escolas municipais de Florianópolis observou que 69\% dos escolares consumem fast foods (hambúrguer/pizza, batatas fritas) pelo menos uma vez na semana, 41\% consomem doces mais de uma vez por semana e somente $32 \%$ consomem frutas e vegetais mais de cinco vezes por semana (COSTA; ASSIS, 2012). O ambiente escolar tem se mostrado adequado para a educação nutricional, pois representa o primeiro grupo social em que as crianças têm contato, depois da família, favorecendo a formação de hábitos, inclusive os alimentares (ROCHA et al., 2002). De acordo com Mondini e Monteiro (1995) a educação nutricional tem sido sugerida como alternativa para mudanças no desequilíbrio do consumo alimentar. A educação nutricional consiste em formar hábitos alimentares adequados, divulgar a importância da nutrição para a manutenção da saúde e acrescentar outros conhecimentos a cerca do tema (LIMA, 1997).

Assim sendo, foi criada a Portaria Interministerial no 1.010, de 8 de maio de 2006, que instituiu as diretrizes para a Promoção da Alimentação Saudável no ambiente escolar (BRASIL, 2006). Segundo o Ministério da Saúde, os professores devem participar das ações de educação em saúde na escola, pois aumentam de forma efetiva a resolutividade e o potencial transformador das ações e, portanto, sua eficácia (BRASIL, 2009). No entanto, muitas vezes os professores não se sentem preparados para atuar com o tema saúde (VIEIRA; CARVALHO, 2002), sendo assim, destaca-se a importância da elaboração de materiais didáticos que auxiliem os educadores no processo de ensino e aprendizagem a trabalharem com seus alunos temas relacionados à saúde, destacando a nutrição.

Neste estudo o estado nutricional e o consumo alimentar de escolares da rede pública municipal de Florianópolis, SC, foi avaliado com o objetivo de elaborar um plano de ação organizado na forma de material didático para a educação nutricional no ambiente escolar. 


\section{MATERIAIS E MÉTODOS}

O presente trabalho foi realizado no período entre março e novembro de 2014. Com o intuito de elaborar um material didático para a educação nutricional de escolares realizou-se um estudo caracterizado como pesquisa-ação (Thiollent 2011). De acordo com a metodologia da pesquisa-ação cumpriu-se as seguintes etapas: definição de problemas e tema, referencial teórico, seminários, linha norteadora e plano de ação.

Para a definição de problemas e temas a serem abordados no material educativo, inicialmente realizou-se a análise do diagnóstico do estado nutricional e do consumo alimentar de uma amostra de 640 escolares de 7 a 10 anos matriculados entre o $2^{\circ}$ e o $5^{\circ}$ ano de nove escolas da rede pública municipal de ensino de Florianópolis.

A amostra utilizada para este diagnóstico foi obtida por conveniência a partir dos escolares participantes do levantamento realizado em 2013, na pesquisa "Desenvolvimento e validação de um sistema de vigilância online para a medida do consumo alimentar e atividade física de escolares de 7 a 10 anos", na qual as atividades extensionistas apresentadas no presente artigo estão relacionadas.

Todas as etapas foram aprovadas pelo Comitê de Ética em Pesquisa com Seres Humanos da Universidade Federal de Santa Catarina (Protocolo número 2250/11) e todos os pais ou responsáveis forneceram consentimento por escrito.

Para o diagnóstico do estado nutricional foram aferidos o peso e a altura, respectivamente, em balança da marca Marte ${ }^{\circledR}$ e estadiômetro da marca Alturaexata ${ }^{\circledR}$. O estado nutricional foi avaliado segundo o Índice de Massa Corporal por idade (WHO, 2006).

Para a avaliação do consumo alimentar foi aplicado o Questionário online do Consumo Alimentar e Atividade Física de Escolares (webCAAFE) nas salas informatizadas das escolas participantes.

O questionário webCAAFE é dividido em três seções: ficha de identificação, alimentação e atividade física (DAVIES et al., 2014). A seção sobre alimentação possui um questionário dividido em seis partes, referente as refeições diárias (café da manhã, lanche da manhã, almoço, lanche da tarde, jantar e lanche da noite). Os itens alimentares são selecionados pelas crianças em uma tela com 32 imagens, incluindo itens saudáveis e não saudáveis (COSTA ${ }^{\mathrm{b}}$ et al., 2012). A partir dos dados coletados calculou-se o percentual de escolares segundo o relato de consumo dos 32 alimentos ou grupos alimentares constantes no Questionário webCAAFE. 
Os trinta e dois itens alimentares foram classificados em alimentos marcadores de alimentação saudável e não saudável de acordo com o Guia Alimentar para População Brasileira (2006).

A partir dos dados obtidos na etapa do diagnóstico foram identificados os principais problemas relacionados à alimentação e ao estado nutricional e assim, os temas trabalhados no material didático foram definidos.

Após a definição dos temas procedeu-se a busca pelo referencial teórico, com o objetivo de auxiliar nas argumentações e delinear a linha de raciocínio para abordagem dos temas de forma coerente para o público alvo e o local de atuação.

Durante toda a busca pelo referencial teórico e elaboração do material educativo foram realizados seminários através de encontros presenciais com a equipe do projeto, com o intuito de centralizar as ideias, delinear a problemática, as ações a serem realizadas e os temas sobre alimentação discutidos no material didático. Com a finalidade de facilitar a comunicação sobre o conhecimento técnico da nutrição pelos educadores e educandos, a linha norteadora empregada durante a elaboração de todo o material didático seguiu uma linguagem de fácil compreensão sem a utilização de termos técnicos da área e com a proposta de atividades lúdico-educativas, visando a melhor compreensão dos escolares a cerca de cada tema abordado.

No plano de ação elaborado foram abordados temas envolvendo conhecimentos básicos sobre alimentação saudável, com o intuito de construir junto aos escolares, de forma lúdica, a importância de hábitos de vida mais saudáveis. O plano de ação foi organizado em um material didático ou cartilha educativa, sendo que cada temática abordada na cartilha está estruturada com um texto de apoio diferenciados para educador e aluno, um exemplo de aula dialogada, seguido de atividades lúdico-educativas.

O texto de apoio para o educador consiste em um resumo sobre cada tema contido na cartilha, com o objetivo de propiciar um conhecimento prévio sobre o tema para o educador, capacitando-o para trabalhar com os alunos. O texto de apoio para o aluno também consiste em um resumo sobre o tema, com o objetivo de subsidiar a elaboração das atividades. Ambos foram construídos seguindo todas as etapas previamente descritas. O exemplo de aula dialogada traz uma forma lúdica de como os educadores podem trabalhar com os escolares sobre cada tema, sendo que o conteúdo da aula dialogada é o mesmo abordado nos textos de apoio.

As atividades lúdicas possuem gabarito e comentários pertinentes. As atividades foram pesquisadas em cartilhas já existentes e livros didáticos do ensino fundamental e a linguagem adaptada aos temas abordados. As atividades possuem diferentes graus de dificuldade, a fim de 
contemplar os variados níveis de cognição, sendo que a escolha das atividades a serem realizadas fica a critério do educador, de acordo com cada turma.

Sugestões de atividades sem gabarito e textos de apoio ao aluno encontram-se em anexo na cartilha, os quais podem ser fotocopiados e entregues aos escolares no decorrer da abordagem de cada tema.

Durante o processo de elaboração do material didático, o mesmo foi revisado por uma pedagoga especialista nas sérias iniciais do ensino fundamental, no intuito de garantir que as atividades propostas estavam de acordo com a capacidade cognitiva de escolares de 7 a 10 anos.

\section{RESULTADOS E ANÁLISES}

\section{Diagnóstico do estado nutricional e do consumo alimentar}

Dos 640 escolares avaliados, 334 (52\%) eram do sexo masculino e 306 (48\%) do sexo feminino. Embora a maioria dos avaliados estivesse com estado nutricional adequado (66\%), elevada prevalência de sobrepeso $(20 \%)$ e obesidade (13\%) foi observada (Figura 1).

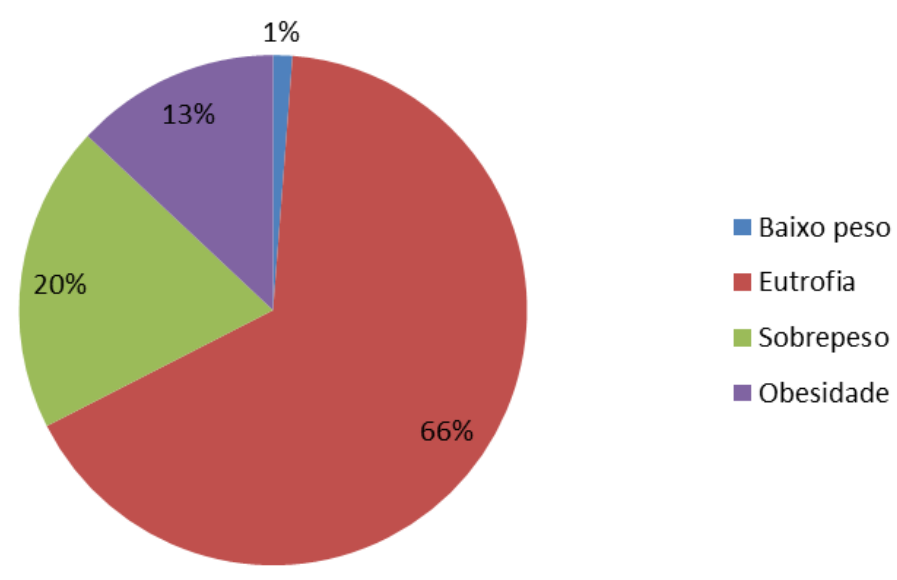

Figura 1. Estado nutricional de 640 escolares da rede pública municipal de Florianópolis-SC, 2014.

Lopes et al., (2010), em estudo realizado com 162 escolares de 7 a 10 anos, encontraram resultados semelhantes, com uma prevalência de 54,9\%,19,8\% e 18,5\%, de eutrofia, sobrepeso e obesidade, respectivamente.

Conforme os resultados encontrados em estudo realizado também no estado de Santa Catarina, com escolares de 6 a 10 anos, as prevalências de sobrepeso e obesidade foram de, respectivamente, $15,4 \%$ e 6,1\% (CORSO et al., 2012). 
De acordo com estudo realizado na cidade de Maringá (PR), com escolares de 6 a 10 anos, a prevalência de excesso de peso foi de $24 \%$, sendo $17 \%$ de sobrepeso e $7 \%$ de obesidade (ROSANELI et al., 2012). Outro estudo realizado em Cruzeiro do Oeste (PR) com a mesma faixa etária, também encontrou uma prevalência semelhante de $24,4 \%$ de excesso de peso, sendo 16,4\% de sobrepeso e 8\% de obesidade (AZAMBUJA et al., 2013).

Segundo estudo que avaliou o estado nutricional de crianças e adolescentes do Nordeste do Brasil, com idade entre 5 e 19 anos, o percentual de sobrepeso encontrado (24\%) também foi semelhante aos observados nesse estudo realizado na região Sul do país (RAMIRES et al., 2014).

Através dos estudos apresentados é possível perceber uma alta prevalência de excesso de peso em crianças e adolescentes em idade escolar, não apresentando grande diferença quando comparadas diferentes regiões do Brasil.

Em relação ao consumo alimentar, observou-se uma elevada prevalência de consumo de alimentos e bebidas não saudáveis e de baixo valor nutricional, ou seja, aqueles cujo consumo não é recomendado pelo Guia Alimentar para a População Brasileira (2006). Na figura 2, estes alimentos estão representados na cor vermelha, com destaque para a prevalência de consumo de biscoito recheado (42\%), suco $(39 \%)$ e refrigerante $(33 \%)$.

Estudo realizado na Escola de Aplicação da Universidade de São Paulo (USP), com 115 escolares de 7 a 10 anos, com o objetivo de identificar os itens alimentares mais representativos no consumo alimentar, também constatou o refrigerante e o suco entre os dez itens mais citados por 49\% dos escolares (HINNING; BERGAMASCHI, 2012). Em outro estudo realizado em São Luís do Maranhão com escolares de 6 a 16 anos, 52,6\% relataram o consumo de biscoito (CONCEIÇÃO et al., 2010).

De acordo com o estudo de Lopes et al., (2010), realizado na cidade de São Paulo, também com escolares de 7 a 10 anos, relacionou o estado nutricional com o consumo de refrigerante, e encontrou associação importante entre o consumo diário de refrigerante e as crianças com sobrepeso, sendo o percentual de sobrepeso de $12,5 \%$ em crianças que consumiam refrigerante diariamente e 3,1\% em crianças que nunca consumiam.

Também foi observado neste estudo baixa prevalência de consumo de alimentos saudáveis, os quais pertencem aos grupos alimentares do Guia Alimentar para a População Brasileira (2006), em que é recomendado o consumo de pelo menos uma porção diária (representados na Figura 2 pela cor azul), dando ênfase as frutas, leite, legumes e verduras, em que apenas $38 \%, 21 \%, 21 \%$ e $17 \%$, respectivamente, dos escolares relataram consumir esses alimentos no dia anterior ao preenchimento do questionário webCAAFE. 
Um estudo de Costa ${ }^{a}$ et al., (2012) encontrou também baixa frequência de consumo de frutas e hortaliças, por parte dos escolares matriculados entre o segundo e o quinto ano da rede pública e privada de ensino de Florianópolis, em que o consumo de frutas e hortaliças, cinco ou mais vezes ao dia, foi relatado por apenas $2,7 \%$ dos escolares, enquanto que $26,6 \%$ não consumiram estes alimentos nenhuma vez ao dia.

Segundo estudo de Bernardo et al., (2012) realizado com escolares da mesma faixa etária do presente estudo, também de Florianópolis, utilizando o Questionário Alimentar do Dia Anterior (QUADA) versão 3, constatou que cerca de 84,9\% dos escolares consumiam frutas, verduras e legumes menos que cinco vezes ao dia e que 67,6\% consumiam refrigerante, doces, salgadinhos em pacote, batata frita, pizza ou hambúrguer duas ou mais vezes ao dia.

Em outro estudo realizado com escolares de Florianópolis, o resultado encontrado seguiu o mesmo padrão, em que a prevalência de consumo de alimentos do tipo fast-foods e doces era maior que o consumo de frutas e vegetais, sendo constatado que 69\% consumiam hambúrguer, pizza e batatas fritas, pelo menos uma vez na semana, $41 \%$ consumiam doces mais de uma vez por semana e apenas $32 \%$ consumiam frutas e vegetais mais de cinco vezes por semana (COSTA; ASSIS, 2012).

Os sucos e refrigerantes se destacaram como dois dos alimentos mais consumidos pelos escolares e o leite entre os alimentos com menor consumo. Estudo realizado no Rio de Janeiro, com alunos do $5^{\circ}$ ano, observou que cerca de $40 \%$ dos escolares consumiam refrigerantes e apenas cerca de 20\% consumiam leite de 2-4 vezes por semana (NOGUEIRA; SICHIERI, 2009).

Observa-se que o consumo alimentar da população em geral, inclusive das crianças, vem se alterando, com uma tendência a substituir alimentos naturais por industrializados. Em um estudo de Levy-Costa e colaboradores (2005), o qual avaliou a disponibilidade domiciliar de alimentos, observou que a caloria média per capita fornecida pelos alimentos variava entre $1800 \mathrm{e}$ $2400 \mathrm{kcal}$, e $28 \%$ das calorias, correspondiam a alimentos de alta densidade energética dentre eles, açúcar e refrigerantes, $18 \%$ das calorias correspondiam as carnes, leite e derivados e ovos e apenas $2,3 \%$ correspondiam às calorias fornecidas pelas frutas, verduras e legumes. 


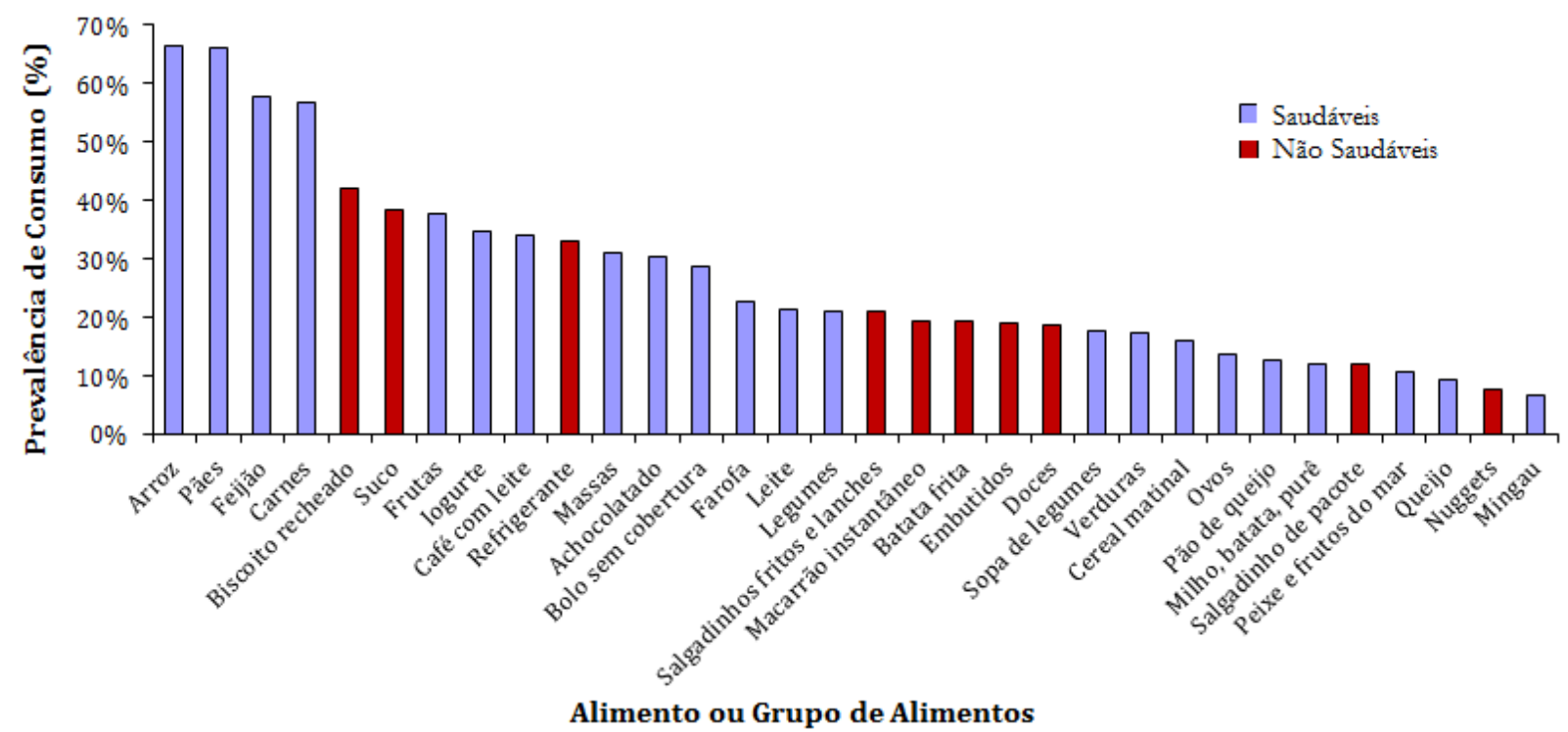

Figura 2. Prevalência de consumo dos 32 itens alimentares do questionário web CAAFE por 640 escolares da rede pública municipal de Florianópolis-SC.

Essas mudanças, observadas nos padrões alimentares, principalmente entre crianças e adolescentes, caracterizada pela redução do consumo de frutas e hortaliças e o aumento do consumo de alimentos industrializados e densamente calóricos, como bolachas recheadas, salgadinhos, doces e refrigerantes, com frequência são associadas ao aumento da prevalência de sobrepeso/obesidade (ENES; SLATER, 2010).

Uma questão que parece influenciar no consumo alimentar de crianças são os meios de comunicação, principalmente a televisão. Foi observado em estudo de Salmom et al. (2006), relação entre a baixa prevalência de consumo de frutas, legumes e verduras e o elevado número de horas em frente à televisão. Em um estudo realizado na cidade de Florianópolis, com a faixa etária de sete a dez anos, observou que as crianças consumiam desde guloseimas a refeições completas em frente à televisão (FIATES et al., 2007).

A influência da televisão no excesso de peso das crianças, não se dá apenas, à substituição da atividade física pela prática de assistir televisão, mas também através dos anúncios publicitários de alimentos de baixo valor nutricional que são transmitidos. Em estudo realizado com escolares, em Florianópolis (SC), 77,5\% referiu que costuma pedir aos pais para comprar os alimentos que são anunciados na televisão e entre os mais pedidos estavam bolachas, flocos de milho açucarados, salgadinhos de pacote, seguidos por guloseimas doces, sucos industrializados, iogurte e refrigerantes (FIATES et al., 2007).

Destaca-se a importância da promoção da alimentação saudável ainda na infância, já que nesta fase da vida as crianças são influenciadas por diversos fatores e estão adquirindo hábitos 
que permanecerão na vida adulta. Mudanças nos hábitos alimentares nessa idade podem prevenir o aparecimento de doenças crônicas na vida adulta, como obesidade, hipertensão, diabetes e doenças cardiovasculares (CRAIGIE et al., 2011).

Em relação aos hábitos alimentares destaca-se o aumento do consumo de açúcares simples, alimentos industrializados e ingestão insuficiente de frutas e hortaliças, resultado semelhante ao encontrado neste estudo. Isso associado à diminuição de atividade física, devido ao maior tempo dedicado às atividades que promovem pouco gasto energético como assistir televisão, usar computador e jogar videogame, também contribui para o ganho de peso excessivo (ENES; SLATER, 2010).

\section{Plano de ação: material didático para a educação nutricional de escolares de 7 a 10 anos}

Após análise e discussão dos resultados encontrados em relação ao estado nutricional e consumo alimentar dos escolares avaliados, obteve-se subsídios para planejar e estruturar uma ação específica para o público estudado.

Dessa forma, a ação proposta foi uma cartilha didática intitulada "Cartilha para educação nutricional de escolares de 7 a 10 anos" desenvolvida para auxiliar os educadores na abordagem da educação nutricional no ambiente escolar durante todo o ano letivo.

Os temas trabalhados na cartilha estão organizados em oito capítulos. Na figura 3, pode ser observado as temáticas abordadas e alguns exemplos de textos de apoio ao professor e ao aluno, aula dialogada e atividades lúdico educativas presentes na cartilha.

Os dois primeiros capítulos abordam os seguintes temas: "Alimentação saudável: os alimentos e as refeições" e os "Grupos alimentares". Estes dois capítulos abordam sobre as recomendações do Guia Alimentar para a População Brasileira (2006), reforçando a importância de se realizar entre cinco e seis refeições diárias e de se consumir alimentos de todos os grupos alimentares tais como o dos cereais, das leguminosas, das frutas, verduras e legumes, dos laticínios, carnes e ovos, gorduras e açúcares.

O terceiro capítulo traz a abordagem sobre a "Origem dos alimentos naturais e industrializados". Destaca-se a importância desse capítulo, quando se analisa o consumo alimentar do grupo estudado, que segue o mesmo padrão de outros estudos anteriormente citados, em que se observa um grande consumo de alimentos industrializados e de baixo valor nutricional e baixo consumo de frutas, legumes e verduras.

Para complementar os capítulos iniciais sobre o consumo alimentar saudável, o quarto capítulo intitulado "Vitaminas e minerais", vem para enfatizar a importância do consumo de 
alimentos ricos em nutrientes para o desenvolvimento saudável, e estimular que as crianças consumam alimentos ricos em vitaminas e minerais, principalmente, frutas, legumes e verduras.

O quinto capítulo é intitulado “Água” e vem trazendo informações sobre as funções e a importância do consumo da mesma. Justifica-se a necessidade desse capítulo devido ao alto consumo de refrigerantes pelos escolares, observado nesse estudo. Estudo de Estima et al., (2011), que avaliou o consumo de bebidas em escolares também encontrou um grande consumo de refrigerantes (28,6\%) enquanto que o consumo de água foi de apenas 9,5\%. Os mesmos autores ainda destacam a importância de ações de educação nutricional que priorizem o consumo de outras bebidas, em relação às bebidas açucaradas, como os refrigerantes.

O sexto capítulo é intitulo "Aprendendo com a horta escolar", e tem como objetivo trazer uma situação prática de estímulo a escolhas alimentares saudáveis, através do trabalho com os alimentos. Segundo Melo et al., (2012) a horta escolar é um elemento capaz de desenvolver temas envolvendo educação alimentar e ambiental no ambiente escolar. Esse capítulo traz a oportunidade de se inter-relacionar os conceitos teóricos apresentados em outros capítulos com o prático encontrado nesse capítulo, auxiliando no processo de ensino aprendizagem.

O sétimo capítulo intitulado de "Publicidade e alimentos", aborda sobre a influência dos anúncios publicitários na alimentação das crianças e sobre a rotulagem nutricional, a qual traz informações nutricionais sobre os alimentos que são anunciados, possibilitando conhecimento mais fidedigno sobre os nutrientes e ingredientes que compõe os alimentos. A importância deste capítulo está no fato de que as crianças atualmente passam muitas horas em frente a televisão e estão expostas a todo momento a propagandas de alimentos, principalmente os de baixo valor nutricional, o que influencia as crianças a consumirem tais alimentos (FIATES et al., 2007).

O oitavo e último capítulo intitulado de "A importância da alimentação escolar" destaca que a alimentação oferecida na escola é saudável, feita especialmente para os escolares, para atender suas demandas energéticas e para fornecer nutrientes importantes para a aprendizagem. Destaca-se a importância de trabalhar esse tema, pois a alimentação escolar também constitui um instrumento de educação nutricional no ambiente escolar. Além disso, Martins et al., (2004), sugere que ações de educação nutricional são necessárias para melhorar a adesão e a aceitação da alimentação escolar.

De acordo com o Marco de Referência de Educação Alimentar e Nutricional para as Políticas Públicas (BRASIL, 2012), o conhecimento deve ser permanente, transdisciplinar, intersetorial e multiprofissional visando promover a prática autônoma e voluntária de hábitos alimentares saudáveis. Dessa forma, destaca-se os educadores como um dos principais grupos alvo que podem propiciar essa educação permanente e transdisciplinar aos escolares. No entanto, Extensio: R. Eletr. de Extensão, ISSN 1807-0221 Florianópolis, v. 13, n. 24, p.49-63, 2016. 
o conhecimento dos educadores sobre o tema é uma condição importante no processo de educação nutricional (BANDURA, 1997).

O desenvolvimento de trabalhos educativos sobre alimentação e nutrição com escolares pode proporcionar conhecimento para os mesmos e consequentemente para seus familiares, contribuindo para favorecer escolhas alimentares mais saudáveis e valorizar a alimentação escolar (OLIVEIRA et al., 2013).

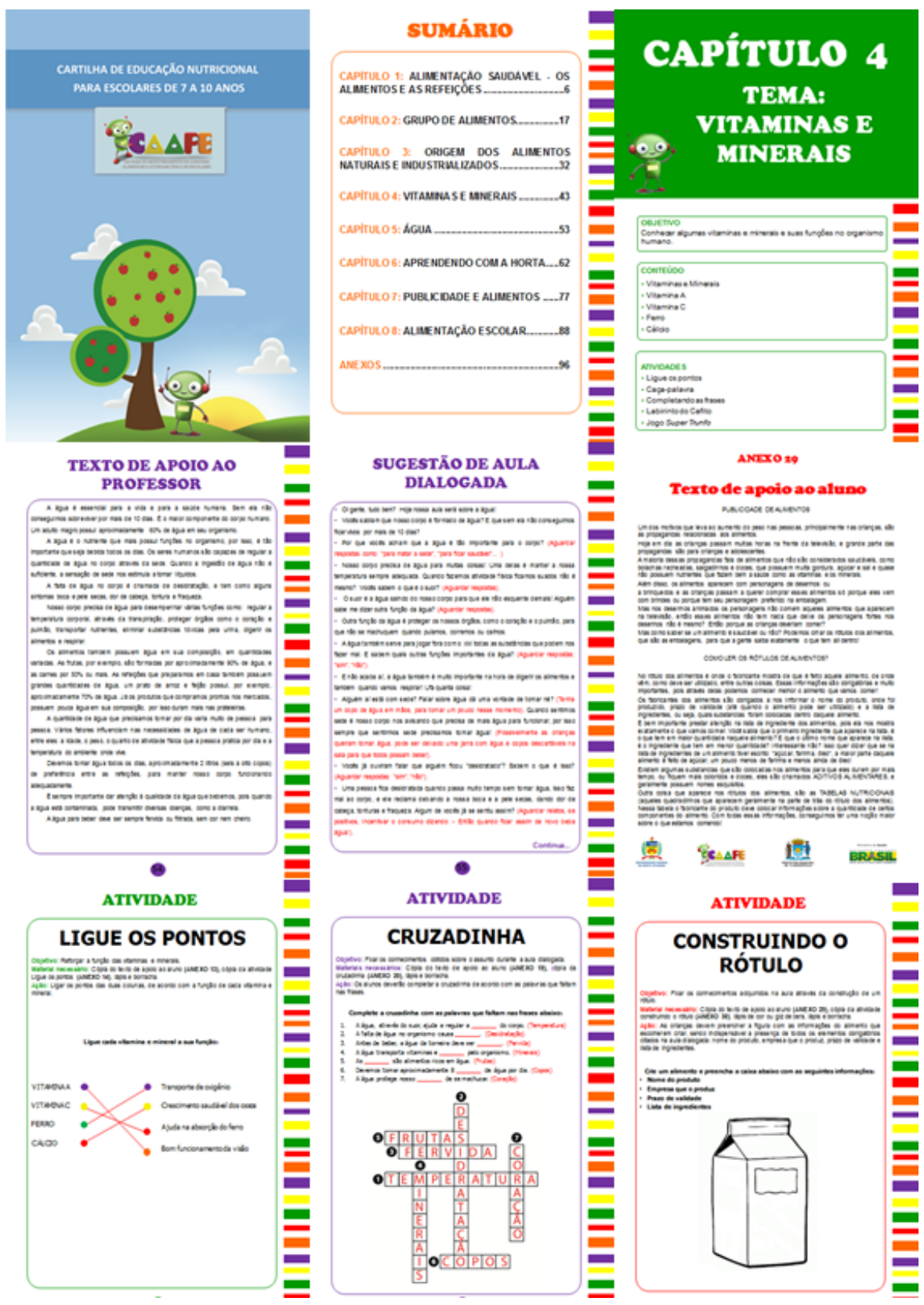

Figura 3. Temas e exemplos de textos de apoio ao professor, sugestão de aula dialogada, texto de apoio ao aluno e atividades lúdico educativas presentes na Cartilha. 
Sendo a educação um fator essencial à vida humana e que ocorre de maneira lenta, gradual e contínua, sugere-se que a educação nutricional ocorra durante toda a trajetória escolar, contribuindo dessa forma para a prevenção de doenças crônicas não transmissíveis principalmente entre as crianças e adolescentes (GOMES, 2013).

Educação nutricional não é uma tarefa simples, pois tem como objetivo mudanças de atitudes, as quais tem relação com conhecimentos empíricos, crenças e valores, e isso envolve certa resistência à adoção de novos hábitos alimentares (BOOG, 2003).

\section{CONSIDERAÇÕES FINAIS}

A infância é um período crucial para a formação de hábitos, e dessa forma ações de educação nutricional nessa fase, podem influenciar nas escolhas alimentares e na adoção de hábitos mais saudáveis que perdurem até a fase adulta.

No presente estudo foi encontrado um grande número de crianças com consumo alimentar inadequado, com excesso de alimentos açucarados e industrializados e baixo consumo de frutas e hortaliças. Além disso, parte significativa das crianças avaliadas apresentou excesso de peso.

Destaca-se a importância deste diagnóstico, antes do planejamento das ações de educação nutricional, visando atividades bem direcionadas aos problemas encontrados no público-alvo. Dessa forma, a Cartilha para a educação nutricional foi organizada com temas específicos que atendessem às demandas do público estudado.

Além disso, a Cartilha elaborada traz como proposta uma educação nutricional que aconteça de forma continuada, já que a mesma será trabalhada pelo educador ao longo dos quatro anos do ensino fundamental, sendo assim mais eficaz do que atividades realizadas de forma pontual.

Como não é possível que o profissional nutricionista, desenvolva atividades de forma direta e contínua com os escolares, ressalta-se a importância do conhecimento dos educadores, para que possam trabalhar tal assunto, uma vez que os mesmos tem um contato mais próximo e possuem maior influência na formação de hábitos das crianças em idade escolar, contribuindo assim na melhora da alimentação e do estado nutricional do público alvo. Espera-se que o material didático elaborado auxilie os educadores no processo de ensino e aprendizagem sobre temas relacionados à alimentação, com o intuito de promover hábitos alimentares saudáveis, visando à redução da prevalência de sobrepeso e obesidade entre os escolares. 
Diagnóstico nutricional e elaboração de material didático para educação nutricional de escolares

\section{AGRADECIMENTOS}

Agradecemos à Pró-Reitoria de Extensão da Universidade Federal de Santa Catarina pela concessão de bolsas ao projeto. Agradecemos também a todas as Escolas Públicas Municipais de Ensino de Florianópolis e a todos os voluntários que participaram e/ou participam do projeto

\section{REFERÊNCIAS}

AZAMBUJA, A.P.O., et al. Prevalência de sobrepeso/obesidade e nível econômico de escolares. Revista Paulista de Pediatria, São Paulo, v.31, n.2, p. 166-171, 2013.

BANDURA, A. Self-Efficacy: the exercise of control. Nova Iorque: Freeman, 1997.

BATISTA FILHO, M., et al. Anemia e obesidade: um paradoxo da transição nutricional brasileira. Cadernos de Saúde Pública, Rio de Janeiro, v. 24, p. 247-257, 2008.

BERNARDO, C.O., et al. Fatores associados ao estado nutricional de escolares de 7 a 10 anos: aspectos sociodemográficos, de consumo alimentar e estado nutricional dos pais. Revista Brasileira de Epidemiologia, São Paulo, v. 15, n. 3, p.651-661, 2012.

BOOG, M.C.F., et al. Utilização de vídeo como estratégia de educação nutricional para adolescentes: "comer... o fruto ou o produto?" Revista de Nutricão, Campinas, v.16, n.3, p.281293, 2003.

BRASIL. Ministério da Saúde. Caderno de Atenção Básica: Saúde na Escola. Brasília, DF, 2009.

BRASIL. Ministério da Saúde. Guia Alimentar para a População Brasileira: promovendo a alimentação saudável, Brasília, DF, 2006.

BRASIL. Ministério do Desenvolvimento Social e Combate à Fome. Marco de referência de educação alimentar e nutricional para as políticas públicas, Brasília, DF, 2012.

BRASIL. Portaria Interministerial MS/MEC n. 1.010, de 08 de maio de 2006. Institui as diretrizes para a promoção da alimentação saudável nas escolas de educação infantil, fundamental e nível médio das redes públicas e privadas, em âmbito nacional. Diário Oficial da União, Brasília, DF, 2006.

CONCEIÇÃO, S.I.O., et al. Consumo alimentar de escolares das redes pública e privada de ensino em São Luís, Maranhão. Revista de Nutrição, Campinas, v. 23, n. 6, p. 993-1004, 2010.

CORSO, A.C.T., et al. Fatores comportamentais associados ao sobrepeso e à obesidade em escolares do Estado de Santa Catarina. Revista Brasileira de Estudos da População, Rio de Janeiro, v. 29, n. 1, p. 117-131, 2012. 
Diagnóstico nutricional e elaboração de material didático para educação nutricional de escolares

COSTA, F.F.; ASSIS, M.A.A . Self-reported physical activity and food intake patterns in schoolchildren aged 7-10 from public and private schools. Revista Brasileira de

Cineantropometria e Desempenho Humano, Florianópolis, v.14, n.5, p. 497-506, 2012.

${ }^{a}$ COSTA, L.C.F., et al. Fatores associados ao consumo adequado de frutas e hortaliças em escolares de Santa Catarina, Brasil. Caderno de Saúde Pública, Rio de Janeiro, v. 28, n. 6, p. 1133-1142, 2012.

${ }^{\mathrm{b}}$ COSTA, F.F., et al . Mudanças no consumo alimentar e atividade física de escolares de Florianópolis, SC, 2002 - 2007. Revista de Saúde Pública, São Paulo, v. 46, supl. 1, p. 117 125, 2012.

CRAIGIE, A.M., et al. Tracking of obesity-related behaviours from childhood to adulthood: a systematic review. Maturitas, v.70, n.3, p.266-284, 2011.

DAVIES, V.F., et al. Qualitative analysis of the contributions of nutritionists to the development of an online instrument for monitoring the food intake of schoolchildren. Journal of Human Nutrition and Dietetics, Florianópolis, p.1-8, 2014.

ENES, C.C.; SLATER, B. Obesidade na adolescência e seus principais fatores determinantes. Revista Brasileira de Epidemiologia, v.13, n.1, p.163-171, 2010.

ESTIMA, C.C.P., et al. Consumo de bebidas e refrigerantes por adolescentes de uma escola pública. Revista Paulista de Pediatria, São Paulo , v.29, n.1, p.41-45, 2011.

FIATES, G.M.R., et al. Television use and food choices of children: qualitative approach. Appetite, v. 50, p.12-18, 2007.

GOMES, D.C. Educação Nutricional: uma necessidade eminente no currículo do Estado de São Paulo. [Monografia]. Universidade Tecnológica Federal do Paraná. Medianeira, 2013.

HINNING, P.F; BERGAMASCHI, D.P. Itens alimentares no consumo alimentar de crianças de 7 a 10 anos. Revista Brasileira de Epidemiologia, São Paulo, v. 15, n. 2, p. 324-334, 2012.

INSTITUTO BRASILEIRO DE GEOGRAFIA E ESTATÍSTICA (IBGE). Pesquisa de Orçamentos Familiares 2008-2009. Antropometria e estado nutricional de crianças, adolescentes e adultos no Brasil. Rio de Janeiro: Instituto Brasileiro de Geografia e Estatística, 2010.

LEVY-COSTA, R.B., et al. Disponibilidade domiciliar de alimentos no Brasil: distribuição e evolução (1974-2003). Revista de Saúde Pública, v.39, n.4, p. 530-540, 2005.

LIMA, E.S. Gênese e constituição da educação alimentar: a instauração da norma. [Tese]. São Paulo: PUC; 1997.

LOPES, P.C.S., et al. Fatores de risco associados à obesidade e sobrepeso em crianças em idade escolar. Revista Brasileira de Enfermagem, Brasília, v. 63, n. 1, p. 73-78, 2010.

MARTINS, R.C.B. et al. Aceitabilidade da Alimentação Escolar no Ensino Público Fundamental. Saúde em Revista, Piracicaba, v.6, n.13, p. 71-78, 2004. 
Diagnóstico nutricional e elaboração de material didático para educação nutricional de escolares

MATOS, S.M.A., et al. Weight gain rate in early childhood and overweight in children 5-11 years old in Salvador, Bahia State, Brazil. Cadernos de Saúde Pública, Rio de Janeiro, v. 27, n. 4, p. 714-722, 2011.

MELO, T. F. T.A., et al. .A horta escolar como estratégia do PIBID na Educação

Ambiental. In: X Jornadas Nacionales y V Congresso Internacional de Enseñanza de la Biología, Córdoba, p. 683-688, 2012.

MONDINI, L.; MONTEIRO, C.A. Mudanças no padrão de alimentação. In: MONTEIRO, C.A. Velhos e novos males da saúde no Brasil: evolução do país e de suas doenças. São Paulo: Hucitec; p.79-89, 1995.

NOGUEIRA, F.A.M.; SICHIERI, R. Associação entre consumo de refrigerantes, sucos e leite, com o índice de massa corporal em escolares da rede pública de Niterói, Rio de Janeiro, Brasil.

Caderno de Saúde Pública, Rio de Janeiro, v.25, n.12, p. 2715-2724, 2009.

OLIVEIRA, J. C., et al. Educação Nutricional com atividade lúdica para escolares da rede municipal de ensino de Curitiba. Cadernos da Escola de Saúde, Curitiba, v.1, p. 150-168, 2013.

RAMIRES, E.K.N.M., et al. Estado nutricional de crianças e adolescentes de um município do semiárido do Nordeste brasileiro. Revista Paulista de Pediatria, São Paulo, v.32, n.3, p.200207, 2014.

ROCHA, D.G., et al. Escola promotora da saúde: uma construção Interdisciplinar e intersetorial. Revista Brasileira de Crescimento e Desenvolvimento Humano, São Paulo, v. 12, n.1, p. 5763, 2002.

ROSANELI, C.F., et al . Avaliação da prevalência e de determinantes nutricionais e sociais do excesso de peso em uma população de escolares: análise transversal em 5.037 crianças. Revista da Associação de Medicina Brasileira, São Paulo, v.58, n.4, p.472-476, 2012.

SALMON, J., et al. Television viewing habits associated with obesity risk factors: a survey of Melbourne schoolchildren. Medical Journal of Australia, v.182, n.2, p.64-72, 2006.

THIOLLENT, M. Metodologia da Pesquisa-ação. São Paulo: Cortez, 2011.

TRICHES, R.M.; GIUGLIANI, E.R.J. Obesidade, práticas alimentares e conhecimentos de nutrição em escolares. Revista de Saúde Pública. Rio de Janeiro, v.39, n.4, p. 541-547, 2005.

VIEIRA, M.L.F.; CARVALHO, M.M.B. O conhecimento de saúde escolar de professores de escolas publicas: uma visão pediátrica e pedagógica. Revista Paulista de Pediatria, São Paulo, v.20, n.5, p.215-223, 2002.

WHO. World Health Organization. Child growth Standards. WHO, 2006. Disponível em: < http://www.who.int/childgrowth/standards/en/>. Acesso em 22/08/2014. 